\title{
Congenital Heart Defects: Early Surgical Correction and Heart Failure - Brief History -
}

\author{
Manuel Chira ${ }^{1}$, Gabriel Samaşca ${ }^{2^{*}}$ and Angela Butnariu ${ }^{3}$ \\ ${ }^{1}$ Heart Institute Cluj-Napoca, Iuliu Hațieganu University of Medicine and Pharmacy Cluj-Napoca, Romania \\ ${ }^{2}$ Department of Immunology, Iuliu Hațieganu University of Medicine and Pharmacy Cluj-Napoca, Romania
} ${ }^{3}$ Department of Pediatrics III, Iuliu Hațieganu University of Medicine and Pharmacy Cluj-Napoca, Romania

\section{Why Early Primary Correction?}

The first successful cardiac surgical procedure was performed by Robert Gross in 1938 in Boston. A 7 year-old patient underwent ligation of the ductus arteriosus [1]. In 1945, Crawford carried out the first correction for coarctation of the aorta in Sweden [2,3].

Technological development that allowed for the introduction of extracorporeal circulation devices led to the beginning of open heart surgery. The first heart surgery using an extracorporeal circulation device was performed by Kirklin. Extracorporeal circulation devices had multiple negative effects on the physiology of the infant and the newborn, so that at the beginning of pediatric heart surgery, the delay of surgical correction was preferred. Initially, these devices required an extremely high priming volume that exposed the child to exanguinotransfusion. Inflammation mediators [4] caused excessive vascular permeability and tissue edema, all the more so as the age of the child was younger at the time of surgery.

At first, anesthesia and intensive care for children were practically non-existent, and diagnosis was made using invasive techniques (cardiac catheterization), which most frequently generated complications. Surgical kits did not contain microsurgical instruments required for working on the fragile heart tissue of the newborn and infant. Thus, a number of palliative procedures were developed, in order to help the patient survive until the performance of surgical correction.

The pathophysiology of congenital heart defects includes 3 main categories. There may be a volume load in which one or both ventricles must pump more blood than normal. This usually happens because of an increased pulmonary blood flow, as a result of a septal defect. There may be a pressure load of one or both ventricles. This can be the result of an obstruction at the outlet of the ventricle. The third possibility is cyanosis that can be secondary to a reduction in pulmonary blood flow or inadequate mixing between the two parallel circulations.

Systemic pulmonary shunt reduces cyanosis by increasing pulmonary blood flow. Although from a conceptual point of view it is an easy procedure, systemic pulmonary shunt can be a challenge for the surgeon. Assessing the size of the shunt depending on the weight of the child is most important. The size of the shunt must take into consideration the subsequent development of the child, but it must not be too large in order to avoid postoperative ventricular volume overload. The Blalock-Taussig shunt was introduced by the surgeon Alfred Blalock and the cardiologist Helen Taussig [5]. The original BT shunt directly connected the sectioned subclavian artery to the right pulmonary artery and was of an adequate size to substitute for the pulmonary blood flow deficiency. In addition, this shunt had growth potential and thus, it could pathophysiologically support the patient for many years. The Waterston shunt is an anastomosis between the ascending aorta and the right pulmonary artery [6]. This shunt has the disadvantage to produce excessive pulmonary blood flow and a distortion of pulmonary arteries.
The Potts shunt consists of the anastomosis of the descending aorta and the left pulmonary artery. This shunt has all the disadvantages of the Waterston shunt and in addition, it is extremely difficult to remove at the time of surgical correction.

The modified BT shunt, which consists of the anastomosis of the arterial brachiocephalic trunk with the right pulmonary artery, by means of a vascular prosthesis, was introduced by deLeval [7]. This shunt was initially performed in the left subclavian artery and the left pulmonary artery through thoracotomy. It has the following advantages: it does not cause pulmonary artery distortion and pulmonary overload.

High blood pressure and flow in pulmonary circulation, most frequently due to a septal defect, may lead in time to pulmonary hypertension and pulmonary vascular disease. In 1950, Muller and Dammann introduced pulmonary artery banding in order to reduce high blood pressure and flow in pulmonary circulation [8]. Like in the case of shunts, banding does not allow for an increase in diameter, so that in time, with the child's growth, this becomes increasingly tight. It can also result in pulmonary artery distortion, so that correction and the removal of banding should be initiated as soon as possible after the regression of pulmonary hypertension.

In 1950, Blalock and Hanlon introduced surgical atrial septectomy for children with transposition of the great vessels with insufficient mixing [9]. Nowadays, this septectomy is an interventional procedure performed through the rupture of the septum with a balloon catheter, according to the procedure described by Rashkind [10,11].

\section{Disadvantages of the Staged Strategy}

Any palliative surgery has a morbidity and mortality risk regardless of the experience of the surgical team. All these interventions may cause pulmonary artery distortion, volume overload of pulmonary circulation, pericardial adhesions that make subsequent surgical correction difficult, and thoracotomy can create a cosmetic and functional disadvantage of the chest, even late scoliosis. Because palliative surgery does not correct congenital heart disease, the consequences of abnormal circulation will persist.

*Corresponding author: Gabriel Samaşca, Department of Immunology, Croitorilor Street, 19-21, Iuliu Hațieganu University of Medicine and Pharmacy, Cluj-Napoca, Romania, E-mail: Gabriel.Samasca@umfcluj.ro

Received January 31, 2013; Accepted April 30, 2013; Published May 05, 2013

Citation: Chira M, Samaşca G, Butnariu A (2013) Congenital Heart Defects: Early Surgical Correction and Heart Failure - Brief History -. J Biomol Res Ther 2: 108. doi:10.4172/2167-7956.1000108

Copyright: (c) 2013 Chira M, et al. This is an open-access article distributed under the terms of the Creative Commons Attribution License, which permits unrestricted use, distribution, and reproduction in any medium, provided the original author and source are credited. 


\section{Advantages of Early Surgical Correction}

The advantages of early surgical correction have been evidenced since the beginning of pediatric heart surgery. In 1952, Lillehei, by introducing cross-circulation [12,13], using a parent of the patient as an oxygenator, succeeded in correcting a number of congenital heart defects with remarkably low mortality and morbidity rates. The great majority of the patients were infants. Because of a parent who had a cerebrovascular accident after providing support by cross-circulation for his child's heart surgery, this technique was abandoned and subsequently, heart surgery began to be performed with an extracorporeal circulation device. Over the next two decades, this was the standard in heart surgery (palliative surgery and then, at older ages, surgical correction). In 1972, Barrath-Boyes reported the first early surgical corrections in infants for a great number of congenital heart defects [14]. BarrathBoyes and later Castaneda $[15,16]$ presented remarkable results in the early surgical correction of cardiac malformations using extracorporeal circulation and deep hypothermic circulatory arrest.

Over the next decade, the controversy between early primary surgical correction and staged correction persisted. An important event in defining the subsequent orientation of pediatric heart surgery regarding the child's age at the time of correction was the first international congress of pediatric heart surgery in Bergamo, 1988, where Kirklin, the most important opponent of early correction, entitled his stand "Orientation of heart surgery towards a very young age" [17], evidencing the advantages of early surgical correction over the staged strategy. Subsequently, the introduction of arterial switch for the transposition of the great vessels and Norwood surgery for hypoplastic left heart syndrome, after prostaglandin treatment for the maintenance of a patent ductus arteriosus, demonstrated once more the advantages of early surgical correction [18].

\section{Specific Advantages of Early Surgical Correction}

During the first year of life, there is a continuous transition of cardiopulmonary circulation and vascular anatomy and physiology towards adult life. Fetal hemoglobin is gradually replaced by adult hemoglobin. Pulmonary resistance dramatically decreases over the first 3 months of life. The right ventricle, which at birth is as thick as the left ventricle, rapidly decreases in thickness and total mass, while the left ventricle increases in thickness and mass [19]. The left ventricle develops through a combination of hyperplasia and hypertrophy. This occurs until the age of 3 months [20,21]. Coronary vascularization loses its capacity of angiogenesis, so that after the neonatal period, ventricular hypertrophy can be associated with low coronary perfusion [22].

Because palliative procedures do not correct heart anatomy, there may be an inefficiency of normal transition to the adult heart. The best example is that of a patient with atrioventricular septal defect undergoing a palliative pulmonary artery banding procedure. The right ventricular pressure remains at systemic level, the relative ventricular shapes are affected: instead of adopting a shape around the left ventricle, the right ventricle will keep its round shape because of the increased pressure from banding. This round shape of the right ventricle will influence the functioning of the tricuspid valve, which has a different physiology from that of the mitral valve, having cords that emerge from the interventricular septum, inducing in time significant tricuspid insufficiency. Thus, the right ventricle will be both volume and pressure overloaded.

The benefits of early surgical correction for pulmonary circulation are obvious. Correction will allow for the transition towards adult pulmonary circulation in the first year of life [23]. Pulmonary vascular resistance rapidly decreases over the first months of life and alveolar and pulmonary vascularization growth will be normal. If pulmonary arterial pressure and flow are not normal in the first years of life, the risk of developing pulmonary hypertension will occur. The pulmonary arterial intima responds to the stress caused by the increased flow and pressure through thickening and fibrosis, and intimal necrosis may subsequently develop. In general, a patient is considered inoperable if pulmonary resistance is higher than $3 / 4$ of systemic resistance and is fixed, i.e. it does not respond to oxygen administration (resistance higher than 12-15 Wood units). Thus, the patient will become cyanotic, Eisenmenger syndrome being present at this stage.

Pulmonary artery banding was devised for protecting the lungs from pulmonary hypertension. It should reduce pulmonary pressure to at least $50 \%$ of systemic pressure. Regarding cerebral growth, this is important during the first years of life and can be compromised by cardiovascular pathophysiology. The increase in the number of synaptic connections is impressive during the first year of life. The energy metabolism of the brain also undergoes important changes during the first weeks of life, with a spectacular increase in oxygen requirements and ATP metabolism. This allows the developing brain to mobilize energy reserves much more rapidly when needed. The limited number of synapses and the limited energy reserve of the newborn can be compared to the protective strategy of delivering a new computer, with unassembled components and an uncharged battery. Newburger et al. [24] studied postoperative cognitive development in patients with transposition of the great vessels who underwent physiological corrections at different ages. This study demonstrated the presence of a correlation between older age at the time of surgery and deficient postoperative evolution, probably due to the effects of chronic cyanosis on brain development. Chronic congestive failure is frequently reflected in a failure to grow. This affects the development of all organs and particularly, the brain.

In contrast to the effects of chronic cyanosis, the effects of severe acute hypoxia on the brain have been extensively studied. Acute cerebral hypoxia causes a massive release of glutamate and other neurotransmitters. Under the conditions of which neurons have limited energy substrates, their excitation results in cell death. In the developing brain, the highest risk areas in the case of acute hypoxia are the areas with a high density of synaptic connections [25].

Regarding the risk of death, this is lower in early corrections compared to the cumulative risk of palliative surgery, with the intermediate period between palliation and correction, and secondary correction itself.

For the family, the presence of a child with congenital heart disease represents a great stress. The family stress caused by the first palliative surgery, with the period between palliation and correction, when the child does not have a normal cardiac physiology, and the correction itself is much greater than parental stress in the case of early surgical correction, after which the child can return to the family and have a close to normal life. Thus, early surgical correction is an important advantage from the point of view of the family as well. The parents will have a normal child as early as the infancy period, so that their stress and tendency to overprotection will be avoided. From the point of view of society and health care costs, early surgical correction has also proved to involve half the costs of staged correction. 


\section{Preoperative and Operative Heart Failure}

Preoperative volume or pressure load may have a significant impact on postoperative myocardial dysfunction and cardiac output. Ventricular volume load can be determined by intracardiac shunt, ventriculoarterial or atrioventricular valve regurgitation, or aortopulmonary connection. It is important to assess patients with ventricular load, who may be cyanotic or not, may have one or two ventricles, one or two separate ejection pathways, but regardless of the anatomical defect, these patients have congestive heart failure and pulmonary hypertension. Telediastolic volume is increased and contractility is low. Increased volume load and increased telediastolic pressure in the systemic ventricle cause pulmonary edema by the increase of pulmonary venous pressure. Thus, lung compliance is low and air flow resistance is high due to the small airways compressed by the distended vessels [26-28]. On the pulmonary X-ray, in addition to the increased cardiothoracic index, pulmonary circulation is loaded. The ventilation-perfusion discrepancy contributes to the development of an oxygen gradient between the alveoli and the blood system and an increased dead space [29].

The time in which ventricular dysfunction develops is variable, but if surgery for the correction of volume overload is performed during the first 2 years of life, residual ventricular dysfunction is rare [30]. The degree of load volume is also extremely important. For example, a patient with a dilated and dysfunctional ventricle, to which atrioventricular or ventriculoarterial regurgitation is added, is likely to have postoperative systolic ventricular dysfunction. Preoperative treatment with diuretics, vasodilators and inotropic support can be of real help in decreasing volume load and contributes to the reduction of preoperative load volume and possibly to the improvement of ventricular function. If this treatment leads to no improvements, surgery should no longer be delayed. In general, corrective surgery should not be too long delayed, because a spectacular improvement of the preoperative clinical picture cannot be obtained by medical treatment alone, but only after the surgical correction of heart defects.

If the volume load of pulmonary circulation persists, pulmonary vascular changes will begin to appear until pulmonary vascular resistance becomes permanently high [31-33]. The duration until the development of pulmonary hypertension depends on the size of the shunt between the two circulations. The progression of pulmonary hypertension is more rapid when there is both volume and pressure load of pulmonary circulation, as it may happen in a large ventricular septal defect. If pulmonary flow is increased in the absence of high pulmonary pressure, like in the case of an atrial septal defect, pulmonary hypertension develops much later, or does not develop at all.

Ventricular pressure load causes an increase in telediastolic pressure and telesystolic pressure reflecting high contractility and a hyperdynamic status. The maintenance within normal limits of preload, afterload and normal sinus rhythm is important for preventing the decrease of cardiac output and implicitly, the development of coronary hypoperfusion. Because the period until the development of ventricular dysfunction is longer in patients with chronic pressure load compared to patients with chronic volume load, congestive heart failure symptoms are rare and only develop if obstruction is severe and prolonged. An exception to this rule is neonatal obstruction due to aortic stenosis or coarctation of the aorta, in which case left ventricular decompensation may occur very early, even during the first month of life.

The factors that influence ventricular performance include myocardial fiber stretch (preload), loading during myocardial contraction (afterload) and the contractile status of myocardial musculature. Along with heart rate, these factors determine cardiac output and systemic perfusion. Like in adults, oxygen intake is crucial for cellular and organic functioning, particularly during stress such as a disease, physical exercise or postoperative stress. In pediatric patients, particularly during the first months of life, additional stress may unbalance the circulatory system, given that pediatric basal metabolism is much higher than in adults.

Congestive heart failure develops in children with cardiac malformations, like in adults, when myocardial function is deficient and telediastolic volume is increased. These pathophysiological changes manifest as congestive symptoms, such as dyspnea with pulmonary edema or peripheral edemas. However, the symptoms of congestive heart failure may develop in children with congenital malformations even under the conditions of increased cardiac output and moderately high preload. On the X-ray, the signs of heart failure include cardiomegaly, hyperinflation, increased pulmonary circulation or in severe cases, pulmonary edema.

Other symptoms of pediatric congestive heart failure are feeding difficulties, food intolerance, vomiting, failure to grow. At the level of the respiratory system, characteristic are nasal flaring, tachypnea, costal retraction or difficult respiration. The signs of low cardiac output are tachycardia, gallop rhythm, deficient perfusion of the extremities, hepatomegaly that develops much more rapidly than in adults, oliguria, weight gain and less frequently, peripheral edema or even altered states of consciousness. All these signs of heart failure may occur in cardiac malformations with left-to-right shunt, but also in the decompensation stages of a cardiac malformation with right-to-left shunt.

\section{References}

1. Gross RE, Hubbard JP (1939) Surgical ligation of a patent ductus arteriosus Report of first successful case. JAMA 112: 729-731.

2. Crafoord C, Nylin G (1945) Congenital coarctation of the aorta and its surgical treatment. J Thorac Surg 14: 347-361.

3. Gross RE (1945) Surgical correction for coarctation of the aorta. Surgery 18 673-678.

4. Kirklin JK, Westaby S, Blackstone EH, Kirklin JW, Chenoweth DE, et al. (1983) Complement and the damaging effects of cardiopulmonary bypass. J Thorac Cardiovasc Surg 86: 845-857.

5. Blalock A, Taussig HB (1945) The surgical treatment of malformations of the heart in which there is pulmonary stenosis or pulmonary atresia. JAMA 128 189-202.

6. Oelert H, Frank G, Stegmann Th, Luhmer I, Kallfelz HC, Borst HG (1981) Surgical Treatment of Fallot's Tetralogy in Children Under Four Years of Age Cardiovascular Surgery 375-380

7. de Leval MR, McKay R, Jones M, Stark J, MacCartney FJ (1981) Modified Blalock-Taussig shunt. Use of subclavian artery orifice as flow regulator in prosthetic systemic-pulmonary artery shunts. J Thorac Cardiovasc Surg 81: 112-119.

8. Muller WH, Dammann JF (1952) The treatment of certain congenita malformations of the heart by the creation of pulmonic stenosis to reduce pulmonary hypertension and excessive pulmonary blood flow: A preliminary report. Surg Gynecol Obstet 95: 213-219.

9. William HM, Jr. (1951) The Surgical Treatment of Transposition of the Pulmonary Veins. Ann Surg 134: 683-692.

10. Rashkind WJ, Miller WW (1966) Creation of an atrial septal defect without thoracotomy. A palliative approach to complete transposition of the great arteries. JAMA 196: 991-992.

11. Rashkind WJ, Miller WW (1968) Transposition of the great arteries. Results of palliation by balloon atrioseptostomy in thirty-one infants. Circulation 38 : 453-462. 
Citation: Chira M, Samaşca G, Butnariu A (2013) Congenital Heart Defects: Early Surgical Correction and Heart Failure - Brief History -. J Biomol Res Ther 2: 108. doi:10.4172/2167-7956.1000108

12. Warden HE, Cohen M, Read RC, Lillehei CW (1954) Controlled cross circulation for open intracardiac surgery: physiologic studies and results of creation and closure of ventricular septal defects. J Thorac Surg 28: 331-341.

13. Lillehei CW, Cohen M, Warden HE, Ziegler NR, Varco RL (1955) The results of direct vision closure of ventricular septal defects in eight patients by means of controlled cross circulation. Surg Gynecol Obstet 101: 446-466.

14. Taylor RW (1981) Heart Disease in Infancy. JRSM 74: 471

15. Castaneda AR, Lamberti J, Sade RM, Williams RG, Nadas AS (1974) Openheart surgery in the first three months of life. J Thorac Cardiovasc Surg 68: 719-731.

16. Castaneda AR (1994) Cardiac Surgery of the Neonate and Infant. Philadelphia, WB Saunders.

17. Kirklin JW (1989) The movement of cardiac surgery to the very young. In Crup G, Parenzan L, Anderson RH (eds). Perspectives in Pediatric Cardiology. Mt Kisco, NY, Futura Publishing Co 3-22.

18. Castaneda AR, Norwood WI, Jonas RA, Colan SD, Sanders SP, et al. (1984) Transposition of the great arteries and intact ventricular septum: Anatomical repair in the neonate. Ann Thorac Surg 5: 438-443.

19. Emery JL, Mithal A (1961) Weights of cardiac ventricles at and after birth. Br Heart J 23: 313-316.

20. Zak R (1974) Development and proliferative capacity of cardiac muscle cells. Circ Res 35: 17-26.

21. Anversa P, Olivetti G, Loud AV (1980) Morphometric study of early postnata development in the left and right ventricular myocardium of the rat. I. Hypertrophy, hyperplasia and binucleation of myocytes. Circ Res 46: 495-502.

22. Flanagan MF, Fujii AM, Colan SD, Flanagan RG, Lock JE (1991) Myocardia angiogenesis and coronary perfusion in left ventricular pressure overload hypertrophy in the young lamb: Evidence for inhibition with chronic protamine administration. Circ Res 68: 1458-1470.
23. Reid LM (1984) Lung growth in health and disease. Br J Dis Chest 78: 113-134.

24. Newburger JW, Silbert AR, Buckley LP, Fyler DC (1984) Cognitive function and age at repair of transposition of the great arteries in children. $\mathrm{N}$ Engl J Med 310: 1495-1499.

25. Greenamyre T, Penney JB, Young AB, Hudson C, Silverstein FS, et al. (1987) Evidence for transient perinatal glutamatergic innervation of globus pallidus. $J$ Neurosci 7: 1022-1030.

26. Howlett G (1972) Lung mechanics in normal infants and infants with congenital heart disease. Arch Dis Child 47: 707-715.

27. Bancalari E, Jesse MJ, Gelband H, Garcia O (1977) Lung mechanics in congenital heart disease with increased and decreased pulmonary blood flow. J Pediatr 90: 192-195.

28. Lees MH, Way RC, Ross BB (1967) Ventilation and respiratory gas transfer of infants with increased pulmonary blood flow. Pediatrics 40: 259-271.

29. Levin AR, Ho E, Auld PA (1973) Alveolar-arterial oxygen gradients in infants and children with left-to-right shunts. J Pediatr 83: 979-987.

30. Graham TP (1991) Ventricular performance in congenital heart disease Circulation 84: 2259-2274.

31. Rabinovitch M, Haworth SG, Castaneda AR, Nadas AS, Reid LM (1978) Lung biopsy in congenital heart disease: a morphometric approach to pulmonary vascular disease. Circulation 58: 1107-1122.

32. Hoffman JIE, Rudolph AM, Heymann MA (1981) Pulmonary vascular disease with congenital heart lesions: pathologic features and causes. Circulation 64 873-877.

33. Heath D, Edwards JE (1958) The pathology of hypersensitive pulmonary vascular disease: a description of six grades of structural changes in the pulmonary arteries with special reference to congenital cardiac septal defects. Circulation 18: 533-547. 